\title{
FORAMINÍFEROS BENTÔNICOS COMO INDICADORES BIOESTRATIGRÁFICOS NO QUATERNÁRIO SUPERIOR DA BACIA DE CAMPOS
}

\author{
VALQUÍRIA PORFÍRIO BARBOSA \\ Fundação Gorceix, CENPES, Avenida Horácio Macedo, 950, 21941-915, Rio de Janeiro, RJ, Brasil. \\ valquiriapb.gorceix@petrobras.com.br
}

\begin{abstract}
BENTHIC FORAMINIFERA AS BIOSTRATIGRAPHIC INDICATORS ON UPPER QUATERNARY OF CAMPOS BASIN. Benthic foraminifera are very useful as environmental indicators but provide less resolution concerning the biostratigraphy due to their usual long ranges. Nevertheless, there is a general consensus that the ecological sensibility of particular bathyal assemblages is an important framework to the time constraint. This study aims the proposition of a biostratigraphic zonation to the Upper Quaternary of Campos Basin, using the distribution and frequency patterns of benthic foraminifera as indicators of the major paleoclimatic events related to the glacial and interglacial stages of the last Quaternary glacial cycle. For this purpose, 64 samples from two piston cores drilled on the continental slope of Campos Basin, southwestern Brazil were analyzed. Three benthic biozones were recognized: Cibicidoides pachyderma, Nonionella auris and Gyroidinoides nitidula, which show a good correlation between studied cores and with the international ecozones $\mathrm{X}, \mathrm{Y}$ and $\mathrm{Z}$.
\end{abstract}

Key words: benthic foraminifera, biostratigraphy, upper Quaternary, Campos Basin.

\begin{abstract}
RESUMO - Apesar da menor utilização de foraminíferos bentônicos em bioestratigrafia, muitos bioeventos têm sido reconhecidos como datuns estratigráficos e empregados em correlações bioestratigráficas, sobretudo as de caráter local. Com esse intuito, 64 amostras provenientes de dois testemunhos a pistão coletados no talude continental da bacia de Campos foram submetidas a análises paleontológicas. Como resultado, propõe-se um zoneamento bioestratigráfico local para o Quaternário superior da bacia de Campos por meio de bioeventos expressos no comportamento dos padrões de distribuição e das tendências de frequência das associações de foraminíferos bentônicos batiais, os quais refletem os principais eventos paleoclimáticos relacionados ao último ciclo glacial quaternário. Três biozonas foram identificadas: Cibicidoides pachyderma, Nonionella auris e Gyroidinoides nitidula, as quais mostraram boa correlação entre as seções estudadas e com as ecozonas internacionais de foraminíferos planctônicos W, Y e Z.
\end{abstract}

Palavras-chave: foraminíferos bentônicos, bioestratigrafia, Quaternário superior, bacia de Campos.

\section{INTRODUÇÃO}

O período Quaternário abrange aproximadamente os últimos 2,6 Ma da história geológica da Terra e subdivide-se em duas épocas, Pleistoceno ( 2,6 - 0,01 Ma) e Holoceno ( 0,012 $\mathrm{Ma}$ - Recente). O início do Pleistoceno, atualmente relacionado ao estágio Gelasiano (Gibbard \& Cohen, 2008), é marcado, fundamentalmente, por profundas mudanças no sistema climático da Terra, caracterizadas por contínuas fases de resfriamento que culminaram em uma série de glaciações. $\mathrm{O}$ Holoceno, por sua vez, é marcado, essencialmente, pelo recuo das geleiras pleistocênicas e por uma consequente fase de aquecimento climático e o início do atual interglacial.

O efeito destas mudanças climáticas durante o Quaternário se refletiu de forma diferenciada nas diferentes partes do mundo, podendo ser representado tanto por condições de maior ou menor umidade, como por mudanças de temperatura. A periodicidade destas alterações climáticas também se modificou ao longo do Quaternário, o que culminou com um registro geológico composto por sedimentos depositados sob condições ambientais glaciais, periglaciais e temperadas que, de forma particular, exerceram forte influência sobre as condições físicas, químicas e biológicas dos oceanos e dos continentes.

Neste contexto, apesar do registro continental das glaciações quaternárias e suas evidências terem fornecido inegável contribuição para a reconstrução da história geológica do Quaternário, e terem sido laboriosamente compilados por técnicas estratigráficas clássicas, tanto na Europa quanto na América do Norte, seu registro sedimentar ainda é incompleto, impossibilitando a construção de um modelo de amplitude temporal satisfatório da evolução destes eventos. Assim, a busca por evidências deposicionais mais completas redirecionou as pesquisas neste campo para os depósitos marinhos, nos quais indiscutivelmente o registro destes eventos está melhor representado em função de sua preservação quase contínua. Desde então, muitos estudos de natureza paleoambiental e estratigráfica foram desenvolvidos a 
partir da coleta de testemunhos de mar profundo, destacando-se, em particular, a contribuição dos diversos indicadores biológicos na caracterização e no posicionamento cronoestratigráfico destes eventos.

A utilização de foraminíferos em análises bioestratigráficas estende-se do Paleozóico ao Cenozóico, sendo reconhecidamente um dos grupos de maior aplicabilidade e precisão, principalmente para o Mesozóico e para o Cenozóico. Nos estudos bioestratigráficos convencionais a biocronologia é primariamente condicionada pela existência de eventos evolucionários irreversíveis, como surgimentos e extinções de espécies. Estes eventos fornecem uma boa resolução estratigráfica para o Mesozóico, Paleógeno e Neógeno, porém para o Quaternário a resolução é menor, devido à escassez de marcos evolucionários desta natureza, principalmente em função do curto espaço de tempo decorrido. De acordo com Berggren et al. (1995), atualmente são reconhecidas apenas as biozonas Pt1 e PL6, além da porção superior da biozona PL5 que se estende ao Plioceno.

Isto induziu a procura de outras formas de subdivisão estratigráfica para este período, entre as quais se destaca a utilização da sensibilidade ecológica dos foraminíferos, atualmente um dos critérios mais empregados, especialmente para o Quaternário superior. Assim, partindo de estudos desta natureza realizados em depósitos do Oceano Atlântico Equatorial, do Mar do Caribe e do Golfo do México, Ericsson \& Wollin (1956a,b), construíram curvas climáticas características e de fácil reprodução para o período Quaternário, baseando-se na sensibilidade à temperatura do plexo Globorotalia menardii, expressa em mudanças significativas de frequência deste grupo. Utilizando este mesmo princípio, Ericsson \& Wollin (1968) identificaram uma série de zonas climáticas correlatas a períodos quentes e frios, intrinsecamente relacionadas com os estágios glaciais e interglaciais quaternários, designando-as pelas letras $\mathrm{Q}$ a Z, em ordem decrescente de idade (Figura 1).

Em síntese, este zoneamento consiste no estabelecimento de ecozonas representativas de eventos paleoecológicos, traduzidos em aparecimentos e desaparecimentos ou em reduções e aumentos abruptos na frequência de determinadas espécies (eventos migracionais não-evolucionários), como resposta às flutuações climáticas, considerando-se que formas de águas quentes são incorporadas aos depósitos sedimentares durante episódios interglaciais e, formas de águas frias, durante episódios glaciais. A validade e a reprodutibilidade deste zoneamento vêm sendo deste então confirmadas por numerosos trabalhos subsequentes (e.g. Kennett \& Huddlestun, 1972; Douglas, 1973; Shackleton \& Opdyke, 1973; Bé et al., 1976; Keigwin Jr., 1979; Wornardt \& Vail, 1980; Barash et al., 1983; Vergnaud-Grazzini et al., 1983; Schroeder, 1986; Williams et al., 1988; Shackleton, 1987; Locke \& Thunell, 1988; Martin et al., 1990; Martin et al., 1993; Seto, 1995; Vicalvi, 1997; Scott et al., 1998; Kohl et al., 2004), os quais também têm contribuído com a aquisição e implementação de novas ferramentas (e.g. isótopos estáveis de oxigênio, paleomagnetismo, curvas de variação eustática, entre outros), cuja aplicação integrada mostra ser uma potencial ferramenta de reconstrução geológica, paleoclimática e também bioestratigráfica para o Quaternário (Figura 1). No que se refere aos foraminíferos bentônicos, é fato que a sua sensibilidade ecológica é notadamente uma das ferramentas mais utilizadas atualmente tanto na identificação de eventos climáticos e oceanográficos, como de seus efeitos sobre os parâmetros físicos e químicos dos oceanos. Neste contexto, sabe-se que a mesma sensibilidade às variações climáticas e/ou a seus efeitos observada nas formas planctônicas, também é experimentada pelos foraminíferos bentônicos, embora com menor intensidade graças, entre outros fatores, as variações de temperatura no fundo oceânico serem menos bruscas e oscilarem em uma amplitude inferior às da superfície.

Dessa forma, partindo-se do estabelecimento de calibrações e correlações adequadas e do reconhecimento dos parâmetros ecológicos que controlam a distribuição primária das espécies e associações locais de uma determinada sequência, de modo que seja possível selecionar aquelas que primariamente são controladas por variações de temperatura ou por seus efeitos, acredita-se que eventos paleoecológicos expressos em variações de frequência de espécies ou associações de foraminíferos bentônicos, podem vir a ser utilizados como marcos cronoestratigráficos locais. Com base nestes princípios, apresenta-se neste estudo uma proposta de zoneamento bioestratigráfico local com foraminíferos bentônicos para o Quaternário superior da bacia de Campos, realizado a partir da avaliação dos padrões de frequência e distribuição de espécies e/ou associações batiais.

\section{MATERIAL E MÉTODOS}

Foram analisadas para este estudo 64 amostras provenientes de dois testemunhos a pistão (CAM-257 e CAM-275) perfurados pela Agência Nacional do Petróleo (ANP), durante os anos de 1998 e 1999, pelo Navio Oceanográfico Ultratec II, na região do talude continental da bacia de Campos, sob custódia da Petrobras.

A amostragem obedeceu, preferencialmente, intervalos regulares de $10 \mathrm{~cm}$, tendo sido coletados cerca de $10 \mathrm{~cm}^{3} \mathrm{de}$ sedimentos por amostra, que foram posteriormente lavados em peneira de abertura $0,063 \mathrm{~mm}$, secados em estufa a $60^{\circ} \mathrm{C}$, quarteados e triados em lupa binocular. No quarteamento das amostras foi adotada como fração representativa a quantidade mínima de 400 espécimes de foraminíferos bentônicos.

As datações preliminares das seções baseadas em foraminíferos planctônicos foram desenvolvidas por Vicalvi (2001) como parte de estudos internos da Petrobras nas áreas de talude do Quaternário das bacias de Campos e Santos. Estas datações seguem as propostas de zoneamento bioestratigráfico de Ericsson \& Wollin (1968), acrescida de modificações e/ou calibrações apresentadas por Vicalvi (1997), Scott et al. (1998) e Kohl et al. (2004) (Figura 1).

O zoneamento proposto com base nos foraminíferos bentônicos foi estabelecido a partir da avaliação dos padrões de distribuição e das tendências de frequência da associação, considerando-se eventos de presença, ausência, acme e ocorrência significativa de determinadas espécies. Da avaliação do comportamento das frequências foram eleitas biofácies representativas de eventos ecológicos, que posteriormente foram correlacionados com as clássicas ecozonas de foraminíferos planctônicos de Ericsson \& Wollin (1968), através da datação preliminar destas seções realizada por Vicalvi (2001). 


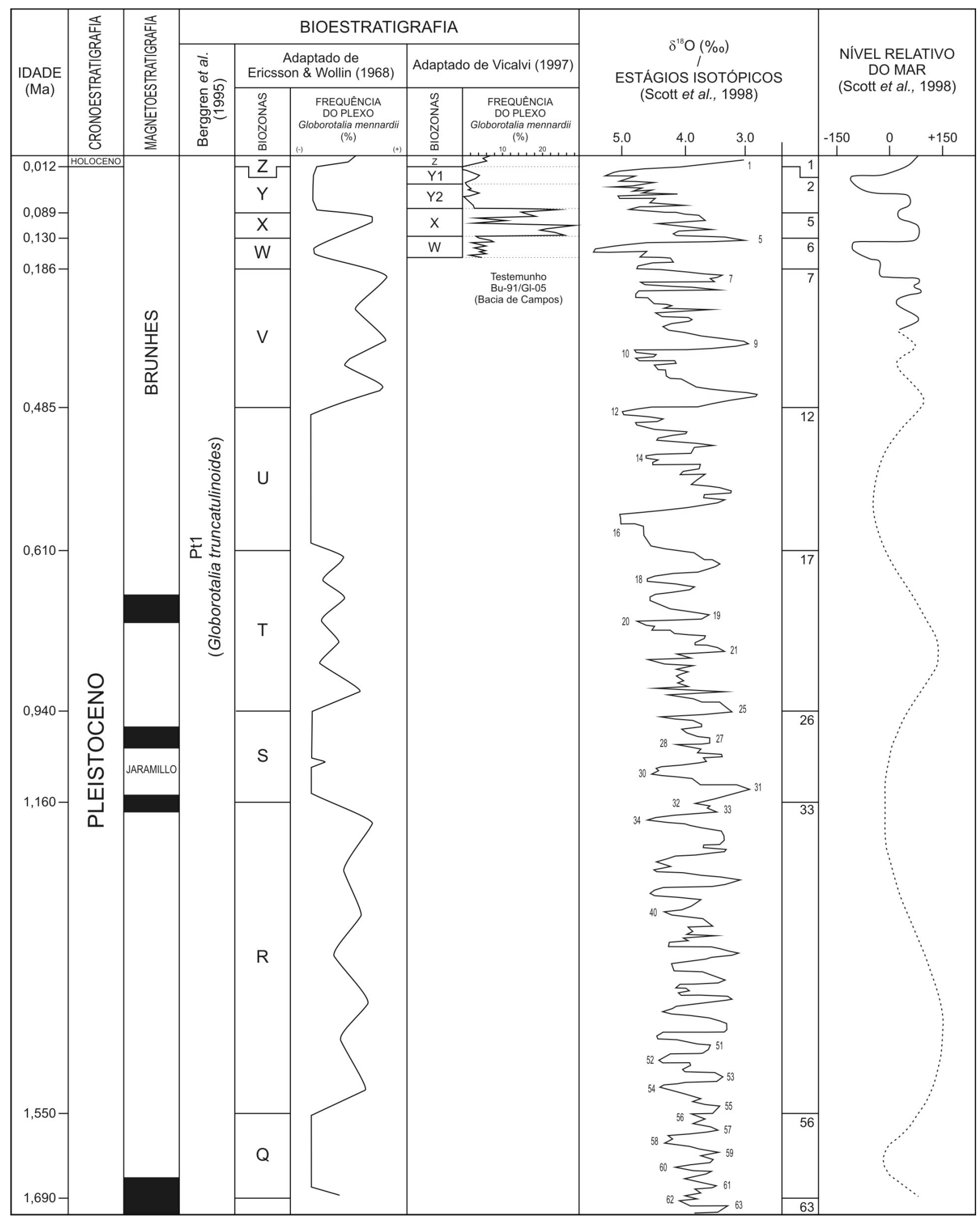

Figura 1. Biocronoestratigrafia do Quaternário superior e principais episódios climáticos definidos por foraminíferos planctônicos, correlacionados com eventos isotópicos de oxigênio e curva global de variação do nível relativo do mar (modificado de Scott et al., 1998).

Figure 1. Upper Quaternary biochronostratigraphy and main climatic events based on planktonic foraminifers, associated to oxygen isotope events and the global relative sea level variation curve (modified from Scott et al., 1998). 
Em síntese, este estudo envolveu as seguintes etapas: (i) levantamento do significado ecológico das espécies; (ii) seleção de espécies exibindo padrões de frequência e distribuição expressivos ao longo dos testemunhos; (iii) seleção de espécies com amplitude e/ou condição de presença/ausência consistentes ou restritos às biozonas de planctônicos; (iv) identificação de espécies com significado paleobatimétrico e/ou paleoclimático; (v) construção de curvas de frequências das espécies selecionadas; (vi) avaliação das tendências exibidas nas curvas de frequência das espécies; (vii) definição de biofácies; (viii) análise qualitativa e quantitativa das biofácies e estabelecimento de correlações com as biozonas de planctônicos.

\section{ÁREA DE ESTUDO}

A área estudada localiza-se na margem continental sudeste do Brasil, aproximadamente entre as coordenadas $22^{\circ} 26^{\prime} 42^{\prime \prime} \mathrm{S}-38^{\circ} 56^{\prime} 17^{\prime \prime} \mathrm{W}$ e $22^{\circ} 33^{\prime} 48^{\prime \prime} \mathrm{S}-39^{\circ} 11^{\prime} 44^{\prime \prime} \mathrm{W}$, com batimetria estimada dentro da zona batial inferior $(\sim 1.200$ $1.700 \mathrm{~m})$ e corresponde à região de talude continental quaternário da bacia de Campos (Figura 2).
A bacia de Campos estende-se ao longo da costa norte do Estado do Rio de Janeiro e sul do Espírito Santo, abrangendo uma área de aproximadamente $100.000 \mathrm{~km}^{2}$. Seus limites geológicos são o Alto de Vitória ao norte e o Alto de Cabo Frio ao sul, os quais separam a bacia das bacias do Espírito Santo e Santos, respectivamente.

Os depósitos quaternários marinhos de águas profundas da bacia de Campos estão inseridos no contexto litoestratigráfico da Formação Ubatuba, essencialmente constituída, segundo Rangel et al. (1994), por pelitos interpostos por arenitos turbidíticos (Formação Carapebus), depositados em ambiente francamente marinho, batial e abissal.

Os sedimentos que compõem as seções estudadas correspondem a depósitos pelágicos (Pleistoceno Superior-Holoceno) constituídos dominantemente por lamitos com níveis e/ou intercalações arenosos, tendo sido registrados oito fácies sedimentares: Areia Fina Bioclástica $(\mathrm{ABs})$; Areia Bioclástica Gradada (ABg); Areia Fina Micácea (AM); Areia Fina Siliciclástica com Bioclastos (ASB); Lama Verde-Oliva Carbonática (LR); Vasa Calcária (VNF); Marga Cinza-Esverdeada (MG); e Lama Calcária (LL) (Figura 3).
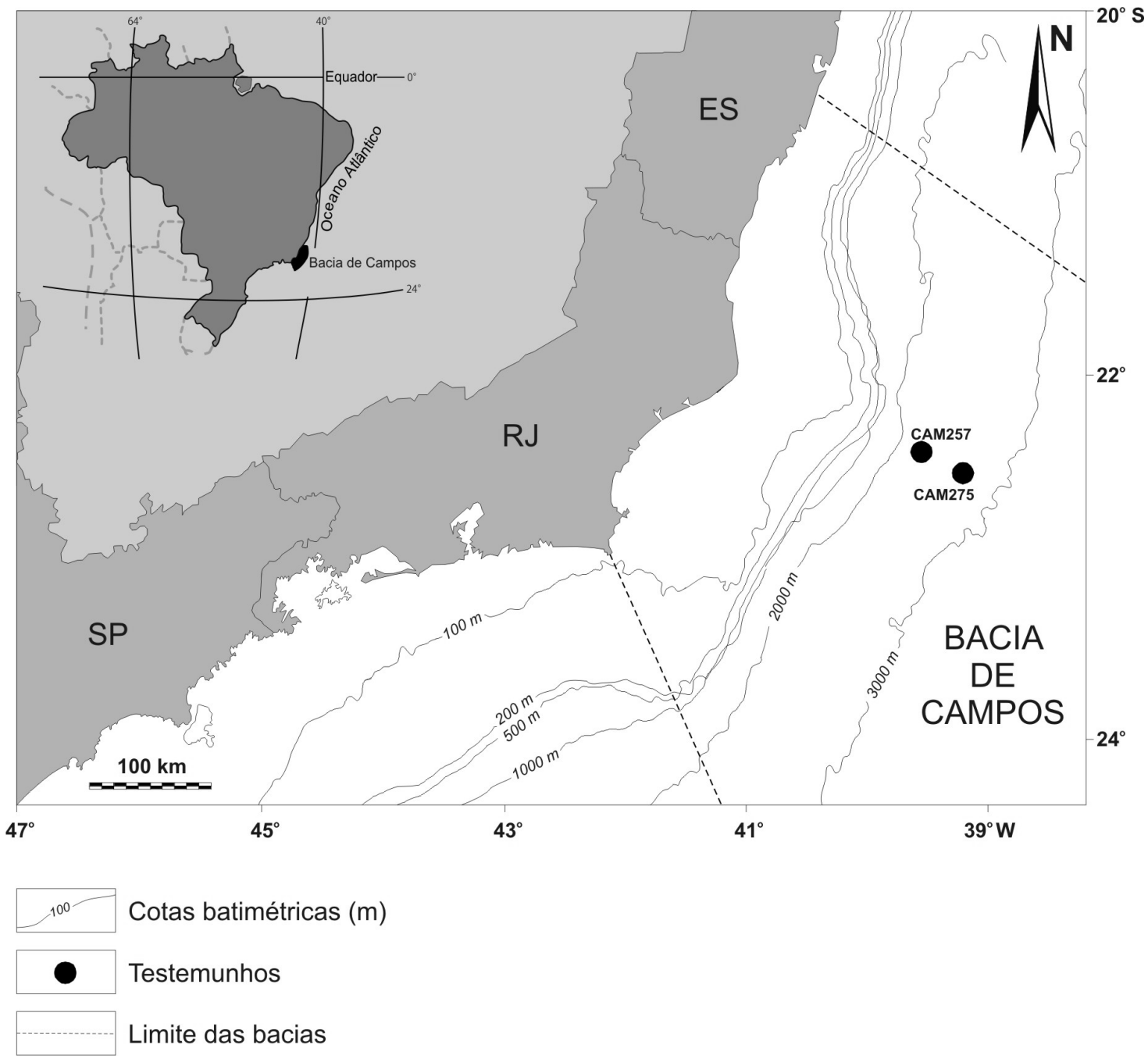

Figura 2. Mapa de localização dos testemunhos estudados.

Figure 2. Location map of studied cores. 


\section{BIOFÁCIES DE FORAMINÍFEROS BENTÔNICOS BATIAIS DO QUATERNÁRIO DA BACIA DE CAMPOS}

Barbosa (2002) estudou a microfauna de foraminíferos bentônicos que compõe a seção quaternária do talude continental da bacia de Campos, no intervalo coberto pelos testemunhos CAM-257 e CAM-275, e registrou uma associação composta por 236 espécies distribuídas em 121 gêneros. Estas espécies foram submetidas a análises qualitativas e quantitativas, visando à identificação dos principais fatores ou parâmetros que controlam a sua distribuição primária (e.g. temperatura, salinidade, batimetria, oxigenação de fundo, concentração de matéria orgânica e nutrientes), de modo a eleger grupos ou associações distintos (biofácies), indicativos destes parâmetros. A partir destas análises foram identificadas duas biofácies, FB1 e FB2, com aparente potencial bioestratigráfico.

Entre os principais constituintes da Biofácies FB1 estão: Ammodiscus gullmarensis, Haplophragmoides bradyi, Haplophragmoides quadratus, Lituotuba lituiformis, Spiroplectella aff. cylindroides, Brizalina subaenariensis, Bulimina echinata, Cibicidoides cicatricosus, Cibicidoides pachyderma, Cibicidoides wuellerstorfi, Gyroidina

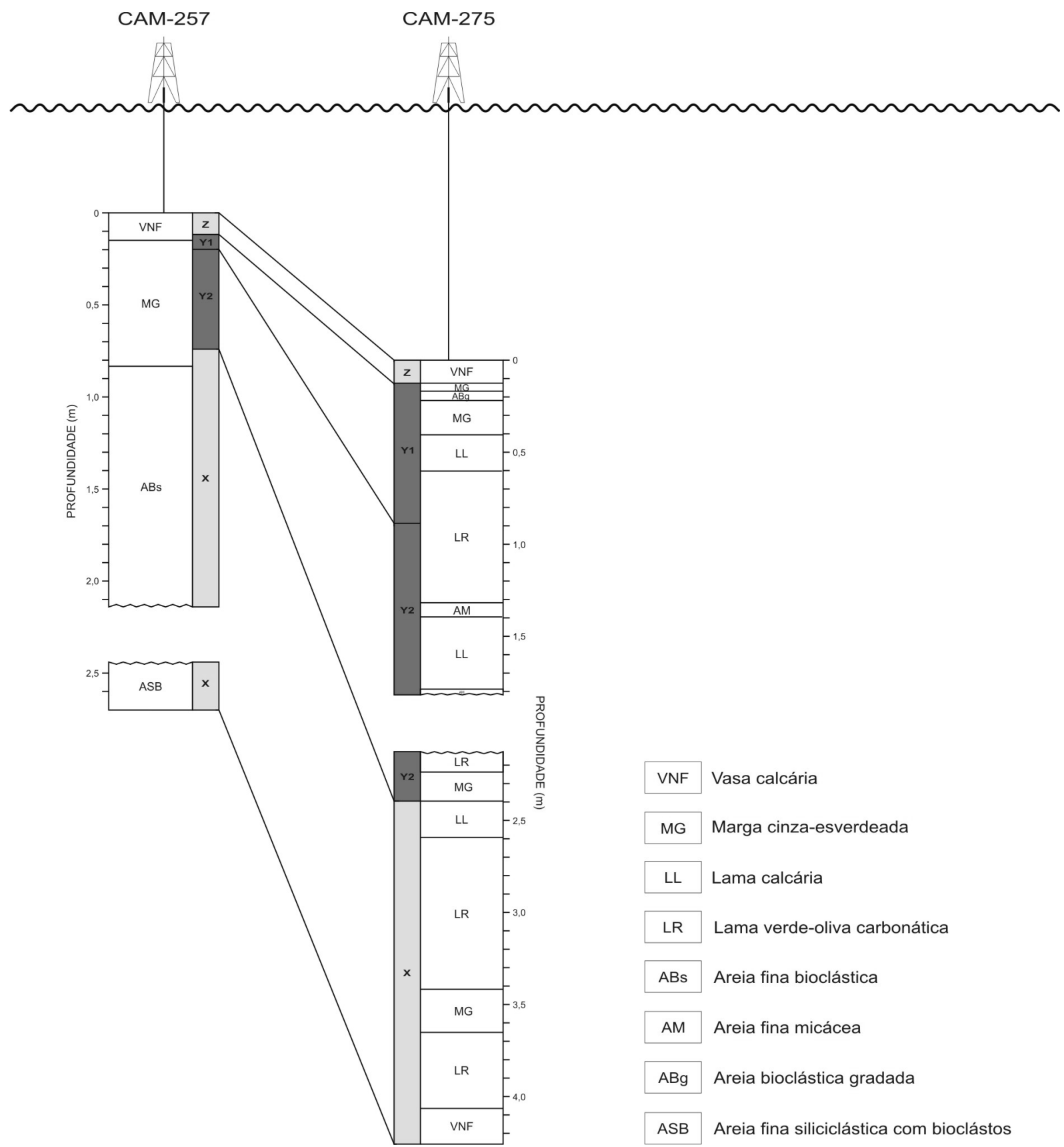

Figura 3. Seção litofaciológica dos testemunhos estudados mostrando a correlação bioestratigráfica entre os poços a partir das zonas de foraminíferos planctônicos de Vicalvi (1997).

Figure 3. Sedimentary section of studied cores, and its biostratigraphic correlation by planktonic foraminifera zones according to Vicalvi (1997). 
lamarckiana, Gyroidinoides nitidula, Islandiella australis, Oridorsalis umbonatus, Oridorsalis variapertura, Osangularia bengalensis, Pyrgoella sphaera, Quinqueloculina venusta, Rutherfordoides calliforniensis, Rhutherfordoides mexicana, Siphouvigerina auberiana, Sigmoilopsis schlumbergeri e Sphaeroidina bulloides (Figura 4).
Observa-se nesta biofácies uma associação com alta riqueza específica, na qual há franco predomínio de morfogrupos epifaunais (tanto entre os aglutinantes, quanto entre os calcários), seguidos, secundariamente, por infaunais de microhábitat raso. De acordo com diversos autores (e.g. Corliss, 1979a,b; Corliss, 1983, 1985; Corliss \& Chen, 1988;
A

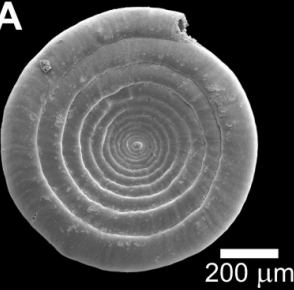

$\mathbf{F}$

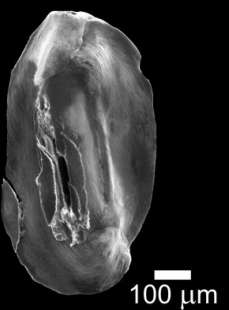

B

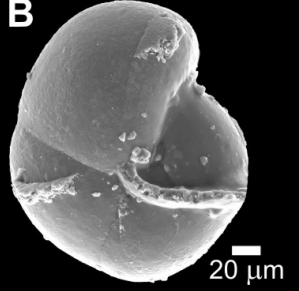

C

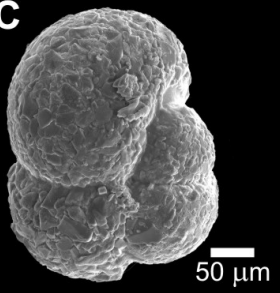

D

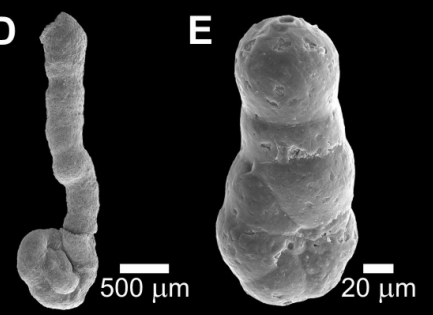

G

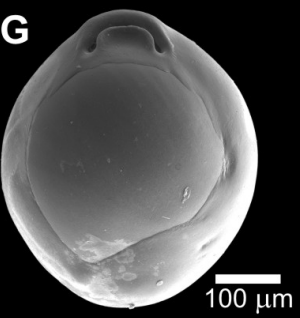

H

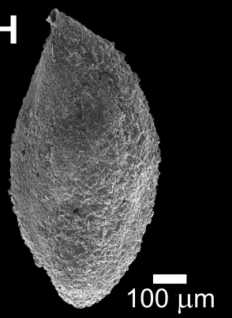

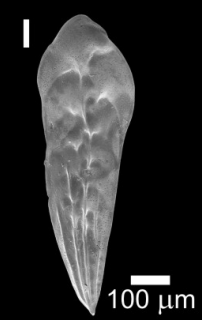
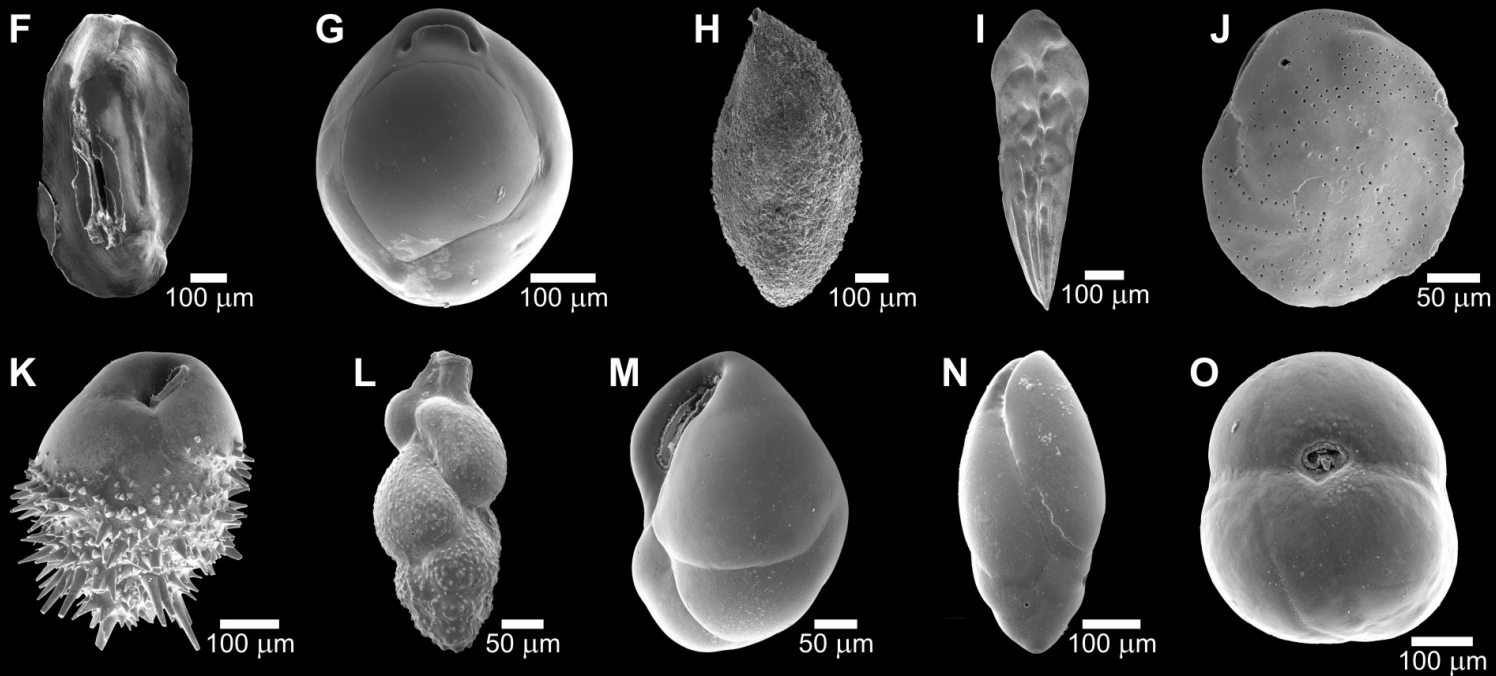

$\mathbf{P}$
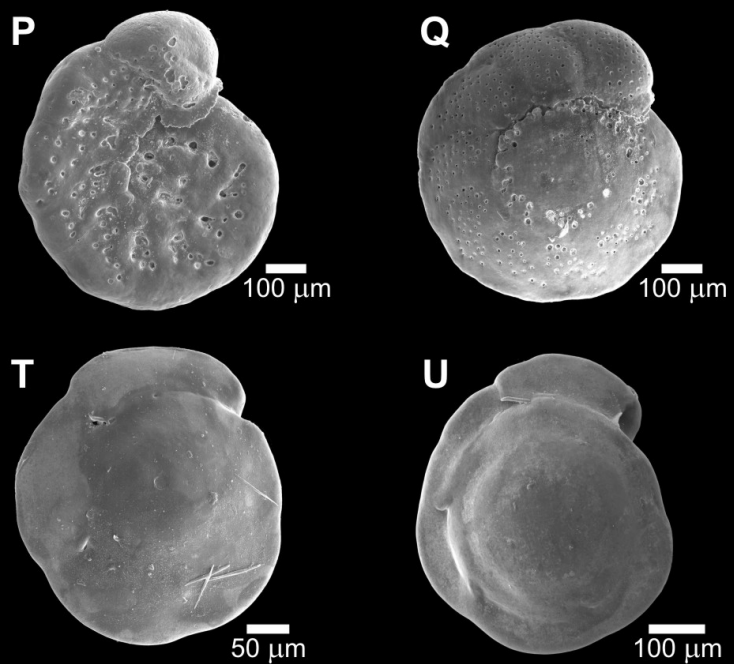

$\mathbf{U}$

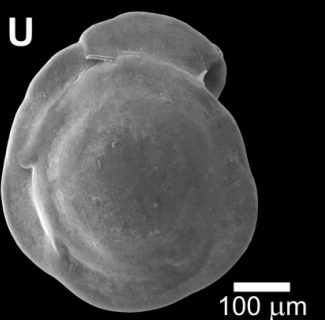

$\mathbf{R}$

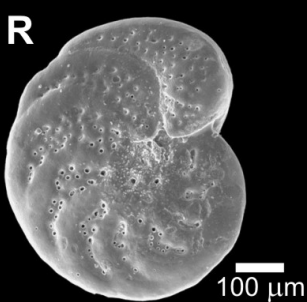

$\mathbf{S}$

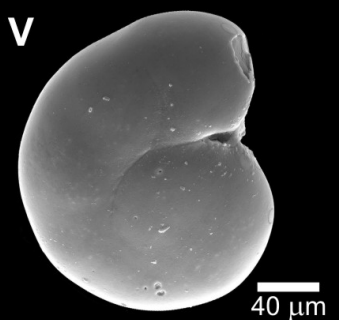

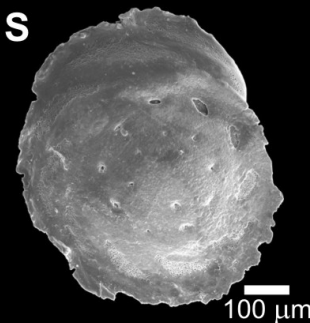

$100 \mu \mathrm{m}$

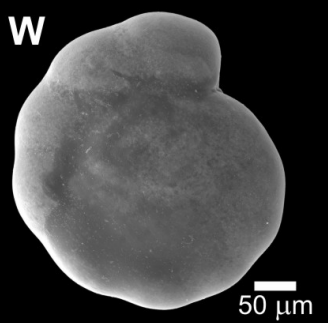

Figura 4. Principais espécies de foraminíferos bentônicos que compõem a Biofácies FB1. A, Ammodiscus gullmarensis; B, Haplophragmoides bradyi; C, Haplophragmoides quadratus; D, Lituotuba lituiformis; E, Spiroplectella aff. Cylindroides; F, Quinqueloculina venusta; G, Pyrgoella sphaera; H, Sigmoilopsis schlumbergeri; I, Brizalina subaenariensis; J, Islandiella australis; K, Bulimina echinata; L, Siphouvigerina auberiana; M, Rutherfordoides californiensis; N, Rutherfordoides mexicana; O, Sphaeroidina bulloides; P, Cibicidoides cicatricosus; Q, Cibicidoides pachyderma; R, Cibicidoides wuellerstorfi; S, Osangularia bengalensis; T, Oridorsalis umbonatus; U, Oridorsalis variapertura; V, Gyroidina lamarckiana; W, Gyroidinoides nitidula.

Figure 4. Most important species of benthic foraminifers of Biofacies FB1. 
Altenbach \& Sarnthein, 1989; Corliss \& Fois, 1991; Barash et al., 2008; Ovsepyan \& Ivanova, 2009), a expressiva presença das formas epifaunais identificadas indica um ambiente bem oxigenado com considerável influxo de matéria orgânica para o fundo, o que também é reforçado por alguns elementos da infauna (e.g. Bulimina echinata e Siphouvigerina auberiana). A composição desta biofácies sugere ainda paleobatimetrias mais elevadas nos intervalos de domínio, além de massas d'água aparentemente mais quentes, evidenciadas, entre outros fatores, por: (i) resultados obtidos a partir da análise de isótopos estáveis de oxigênio em carapaças de Cibicidoides pachyderma e Cibicidoides wuellerstorfi (Barbosa, 2002); e (ii) pela abundância bem marcada destas e de outras espécies, como Gyroidinoides nitidula, Oridorsalis umbonatus, Oridorsalis variapertura, Osangularia bengalensis e Sphaeroidina bulloides, que são comumente associadas a temperaturas de fundo mais quentes (e.g. Mackensen et al., 1985, 1993, 1995; Lear et al., 2002; Martin et al., 2002; Waelbroeck et al., 2002).

A Biofácies FB2, por sua vez, apresenta entre seus principais constituintes: Siphotextularia rolshauseni, Angulogerina angulosa, Bolivina inflata, Bolivina laevigata, Brizalina daggarius, Brizalina pseudogoesii, Bulimina alazanensis, Bulimina elongata, Buliminella elengantissima, Cassidelina complanata, Coriphostoma $\mathrm{cf}$. C. malovensis, Coryphostoma midwayensis, Discorbinella bertheloti, Geminospira bradyi, Gyroidina umbonata, Nonion pacificum, Noninella auris, Paracibicides edomica, Pseudononium granuloumbilicatum, Robertina imperatrix, Sagrina pulchella, Sagrinella subspinescens, Triloculina tricarinata e Triloculinella chiastocytis (Figura 5).

São características dessa biofácies a redução marcante na riqueza específica e a expansão de organismos infaunais, destacando-se aqueles de microhábitat médio a profundo, com domínio de bolivinídeos e buliminídeos, seguidos por um número reduzido de espécies epifaunais de pequeno porte. De acordo com vários trabalhos (e.g. Herguera \& Berger, 1991; Loubere, 1991, 1994, 1996; Sen Gupta \& MachainCastillo, 1993; Kaiho, 1994; Thomas et al., 1995; Jorissen, 1999; Fontanier et al., 2002; Tapia et al., 2008; Licari et al., 2003; Ovsepyan \& Ivanova, 2009), a composição desta biofácies é francamente indicativa de um ambiente com baixa oxigenação de fundo combinada a elevadas concentrações de matéria orgânicas, possivelmente decorrentes de processos de eutrofização no ambiente. A composição desta associação sugere, ainda, uma redução paleobatimétrica e, os dados isotópicos de oxigênio, apontam para águas mais frias.

Os padrões de distribuição e frequência das espécies que compõem estas biofácies parecem sofrer forte influência de condições paleoambientais relacionadas aos episódios de resfriamento e aquecimento do último ciclo glacial quaternário. Esta influência reflete-se principalmente pelo predomínio de uma biofácies em detrimento da outra nos intervalos inferidos por foraminíferos planctônicos como interglaciais (FB1) e glaciais (FB2), respectivamente (e.g. Oppo
\& Fairbanks, 1987; Fairbanks, 1989; Resig \& Cheong, 1997; Bickert \& Wefer, 1999; Ninnemann \& Charles, 2002; Bush \& Fairbanks, 2003; Hayward et al., 2004; Peltier \& Fainbanks, 2006; Piotrowski et al., 2009) (Figuras 6, 7).

A correlação dos intervalos representados por estas biofácies com os intervalos correspondentes às ecozonas de foraminíferos planctônicos mostrou boa correspondência (Figuras 6, 7), o que reforça a indicação do possível controle exercido pelo clima ou de seus efeitos sobre a distribuição das espécies que compõem estas biofácies. Da mesma forma, isso também sugere a possibilidade de que algumas destas espécies ou associações possam constituir ecozonas que venham a ser empregadas como indicadores bioestratigráficos para este intervalo de tempo.

\section{BIOESTRATIGRAFIA}

Com base na avaliação dos padrões de distribuição e frequência das espécies que compõem as biofácies FB1 e FB2, foram estabelecidas três biozonas (ecozonas) de foraminíferos bentônicos para o intervalo estudado: Cibicidoides pachyderma, Nonionella auris e Gyroidinoides nitidula (Figura 8), as quais mostraram boa correlação entre as seções analisadas e com as ecozonas de foraminíferos planctônicos W, Y e Z (Figuras 6, 7).

Zona Gyroidinoides nitidula. Correlata à zona Z de Ericsson $\&$ Woolin (1968), representa um intervalo de aquecimento climático que marca o início do Holoceno. Abrange os intervalos de profundidade 1-12 cm (CAM-257) e 1-17 cm (CAM275) e foi definida considerando-se a amplitude de eventos combinados de acme e de aumentos significativos de frequência de Gyroidinoides nitidula, Ammodiscus gullmarensis, Haplophragmoides quadratus, Pyrgo murrhina e Pyrgoella sphaera. O padrão de distribuição das frequências de Haplophragmoides cf. bradyi, Lituotuba lituiformis, Spiroplectella aff. cylindroides, Cibicidoides wuellerstorfi e Quinqueloculina venusta foram considerados secundariamente na definição desta biozona por também exibirem valores representativos (Figuras 6, 7).

Zona Nonionella auris. Correlata à zona Y de Ericsson \& Woolin (1968), corresponde ao último estágio glacial pleistocênico $(\sim 0,012-0,089 \mathrm{Ma})$, representando, portanto, um intervalo de intenso resfriamento climático. Abrange os intervalos de profundidade de 80-12 cm (CAM-257) e 255-17 cm (CAM-275) e foi definida pela combinação entre intervalos de eventos de acme e de aumentos marcantes de frequência de Nonionella auris, Siphotextularia rolshauseni, Angulogerina angulosa, Bolivina inflata, Bulimina elongata, Nonion pacificum e Paracibicides edomica. Secundariamente, também foram consideradas em sua definição, o comportamento representativo das frequências de Bolivina peseudogoiesii, Bolivina laevigata, Brizalina daggarius, Bulimina alazanensis, Buliminella elegantissina, Cassidelina complanata, Coryphostoma cf. C. malovensis, Coryphostoma midwayensis, Discorbinella 
bertheloti, Geminospira bradyi, Gyroidina umbonata, Pseudononion granuloumbilicatum, Sagrina pulchella, Sagrinella subspinescens, Robertina imperatrix, Triloculina tricarinata e Triloculinella chiastocytis (Figuras 6, 7).

Zona Cibicidoides pachyderma. Correlata à zona X de Ericsson \& Woolin (1968), está relacionada ao último estágio interglacial pleistocênico ( 0,089 - 0,130 Ma), compreenden- do, portanto, um intervalo de aquecimento climático relativamente longo, aparentemente marcado por condições de fundo estáveis, favoráveis ao desenvolvimento e diversificação da microfauna, o que se sugere pela rica associação de foraminíferos bentônicos registrada nesta seção. Abrange os intervalos de profundidade de 80-270 cm (CAM-257) e 255-423 cm (CAM-275) e em sua definição foram considera-
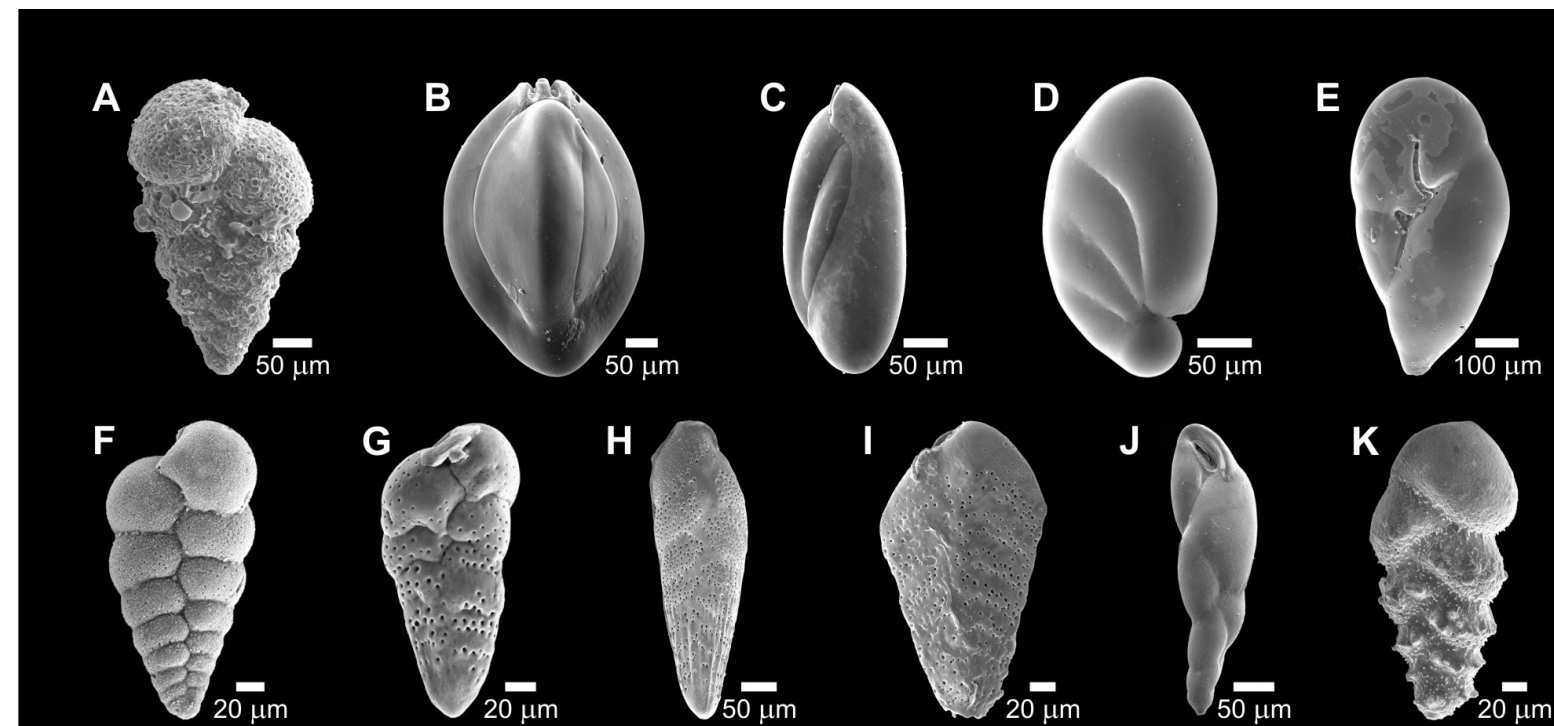

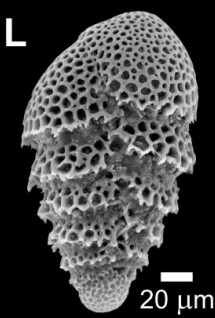

M

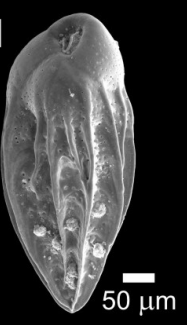

Q
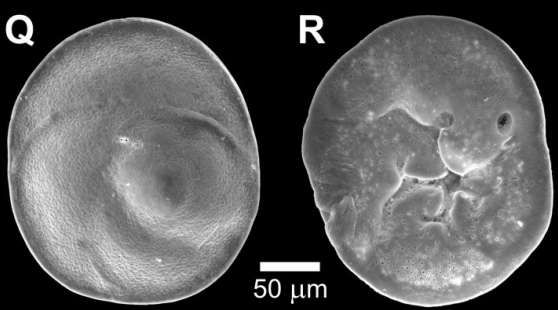

$\mathbf{S}$
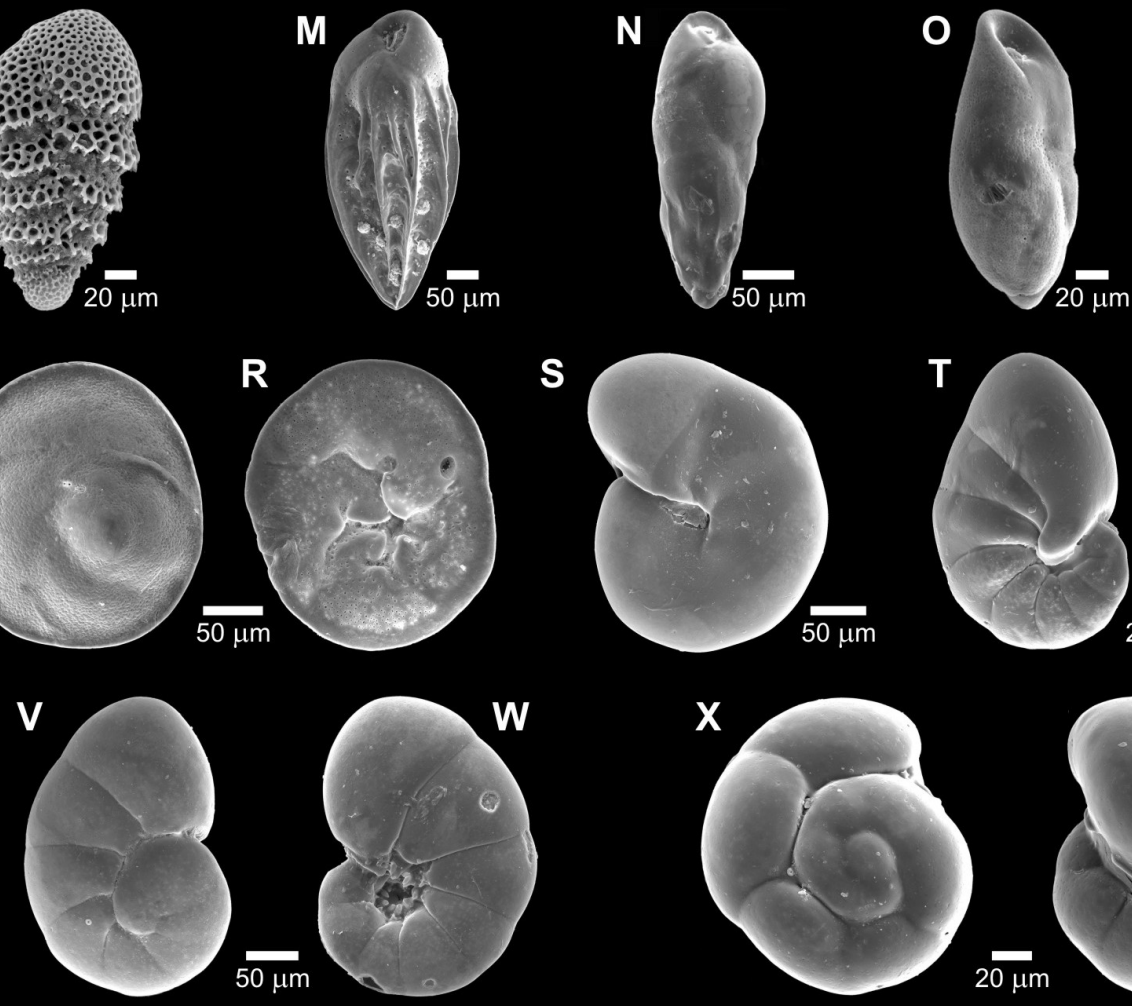

W

$20 \mu \mathrm{m}$
$\mathbf{P}$

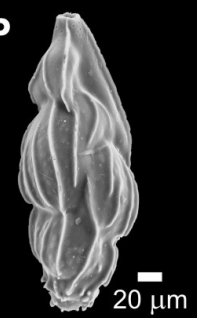

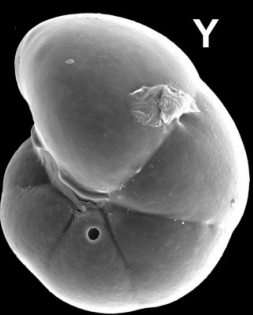

Figura 5. Principais espécies de foraminíferos bentônicos que compõem a Biofácies FB2. A, Siphotextularia rolshauseni; B, Triloculina tricarinata; C, Triloculinella chiastocytis; D, Geminospira bradyi; E, Robertina imperatrix; F, Bolivina inflata; G, Bolivina laevigata; H, Brizalina daggarius; I, Brizalina pseudogoesii; J, Cassidelina complanata; K, Sagrinella subspinescens; L, Sagrina pulchella; M, Bulimina alazanensis; N, Bulimina elongata; O, Buliminella elegantissima; P, Angulogerina angulosa; Q-R, Discorbinella bertheloti; S, Nonion pacificum; T-U, Nonionella auris; V-W, Pseudononion granuloumbilicatum; $\mathbf{X}-\mathbf{Y}$, Gyroidina umbonata.

Figure 5 . Most important species of benthic foraminifers of Biofacies FB2. 


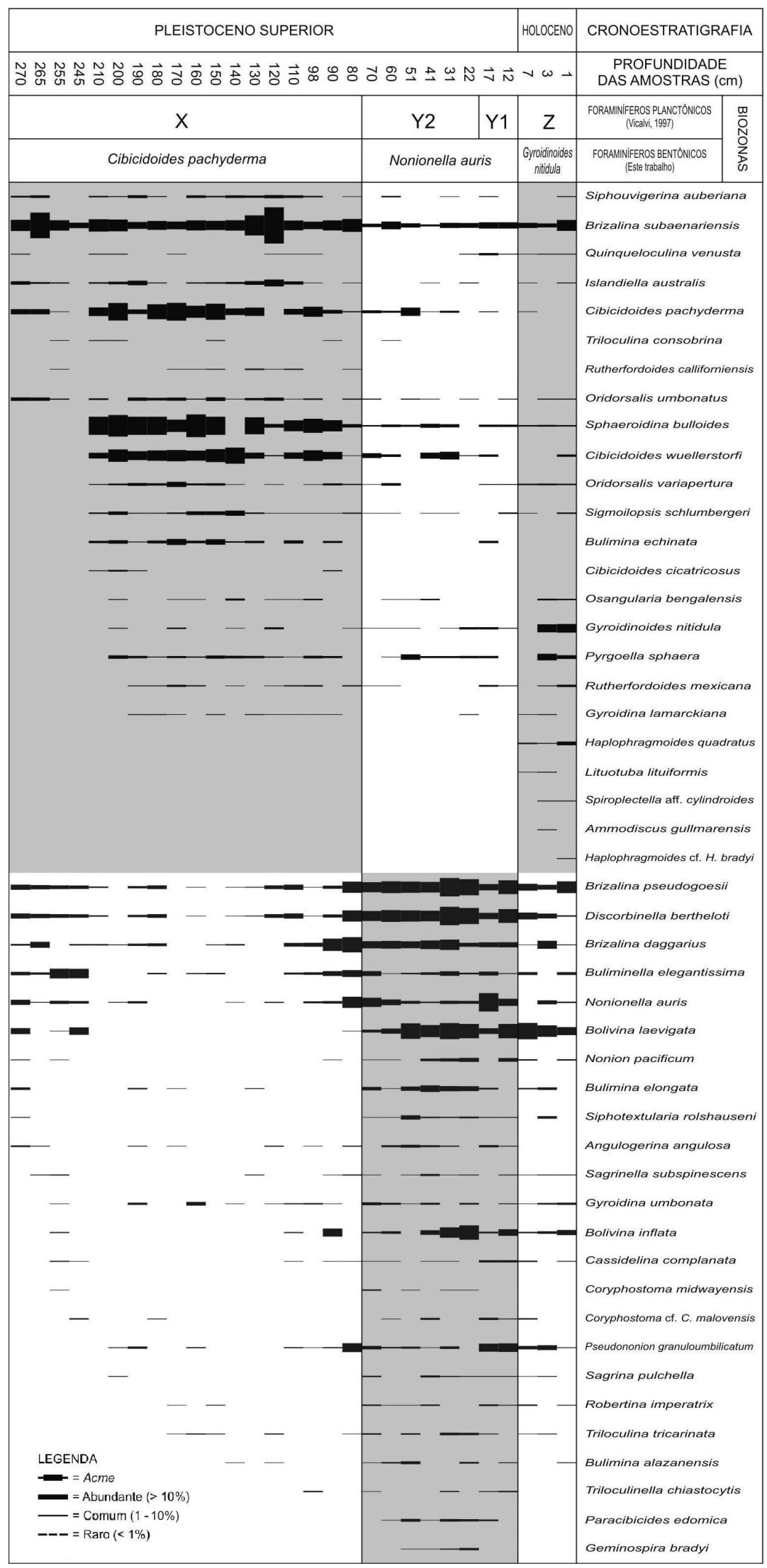

Figura 6. Distribuição das principais espécies de foraminíferos bentônicos representativos das biozonas Gyroidinoides nitidula, Nonionella auris e Cibicidoides pachyderma ao longo do testemunho CAM-257 e sua correlação com o zoneamento de foraminíferos planctônicos.

Figure 6. Distribution of selected benthic foraminifera related to Gyroidinoides nitidula, Nonionella auris and Cibicidoides pachyderma biozones along the CAM-257 core and its correlation with zonal scheme of planktonic foraminifera. 


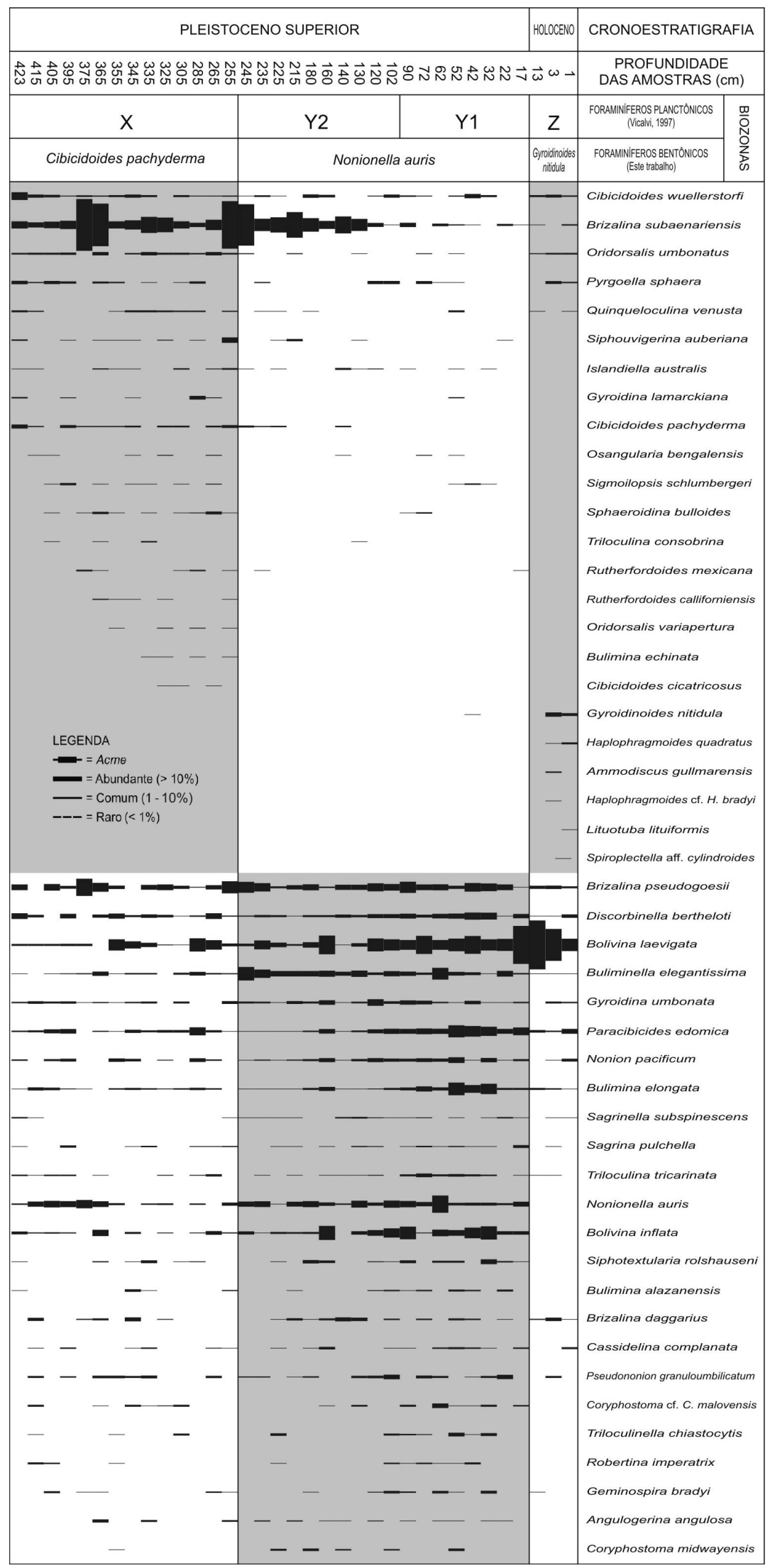

Figura 7. Distribuição das principais espécies de foraminíferos bentônicos representativos das biozonas Gyroidinoides nitidula, Nonionella auris e Cibicidoides pachyderma ao longo do testemunho CAM-275 e sua correlação com o zoneamento de foraminíferos planctônicos.

Figure 7. Distribution of selected benthic foraminifera related to Gyroidinoides nitidula, Nonionella auris and Cibicidoides pachyderma biozones along the CAM-275 core and its correlation with zonal scheme of planktonic foraminifera. 


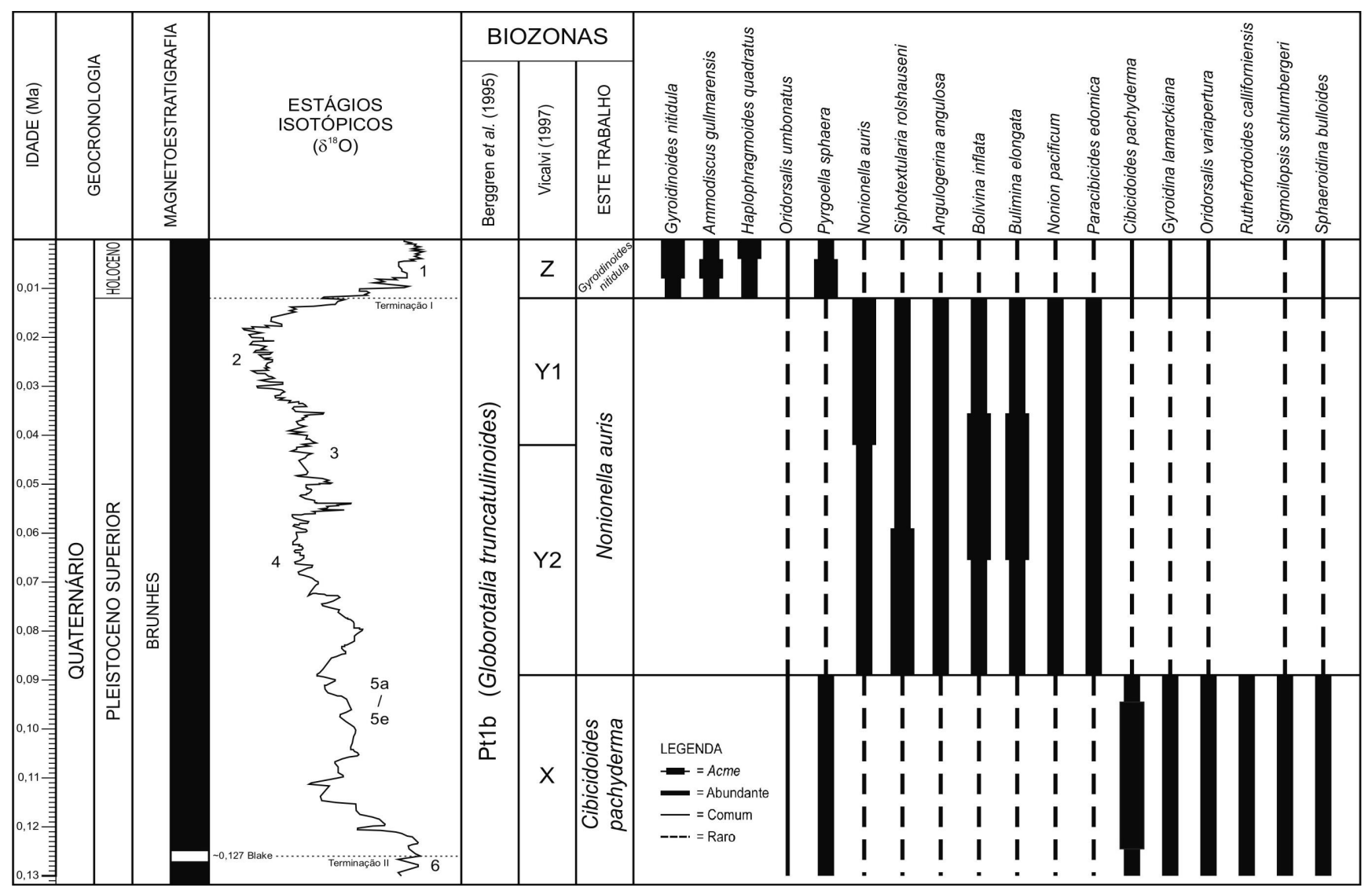

Figura 8. Zoneamento bioestratigráfico para o intervalo Pleistoceno Superior-Holoceno da bacia de Campos com base em foraminíferos bentônicos e sua correlação com os zoneamentos de foraminíferos planctônicos de Berggren et al. (1995) e Vicalvi (1997). Geocronologia, magnetoestratigrafia e estágios isotópicos de oxigênio segundo Gibbard \& Cohen (2008).

Figure 8. Biostratigraphic zonation for the upper Pleistocene-Holocene from Campos Basin, Brazil, based on benthic foraminifera and correlations with the zonal schemes of Berggren et al. (1995) and Vicalvi (1997). Geochronology, magnetostratigraphy and oxygen isotope stages according to Gibbard \& Cohen (2008).

dos eventos combinados de acme e de aumentos expressivos de frequência de Cibicidoides pachyderma, Gyroidina lamarckiana, Oridorsalis variapertura, Pyrgo murrhina, Pyrgoella sphaera, Rutherfordoides calliforniensis, Sigmoilopsis schlumbergeri, Siphouvigerina auberiana e Sphaeroidina bulloides. De forma secundária, também foram considerados na definição desta biozona o comportamento e os valores de frequência de Brizalina subaenariensis, Bulimina echinata, Cibicidoides cicatricosus, Cibicidoides wuellerstorfi, Islandiella australis, Osangularia bengalensis, Quinqueloculina venusta, Rutherfordoides mexicana e Triloculina consobrina (Figuras 6, 7).

\section{CONSIDERAÇÕES FINAIS}

A partir dos resultados obtidos nas análises paleoecológicas e bioestratigráficas dos testemunhos CAM257 e CAM-275 perfurados na região do talude continental da bacia de Campos foi possível estabelecer um zoneamento bioestratigráfico local (ecozonas) para o Quaternário superior, tomando-se como base o padrão de distribuição e as ten- dências de frequência dos componentes de duas biofácies de foraminíferos bentônicos, FB1 e FB2. O comportamento das frequências das espécies que compõem estas biofácies sugere a existência de um controle climático sobre a sua distribuição ao longo das seções estudadas, o qual estaria fortemente relacionado aos episódios de resfriamento e aquecimento (estágios glaciais e interglaciais) do último ciclo glacial quaternário. Da mesma forma, a boa correspondência nas correlações entre o zoneamento de foraminíferos bentônicos obtido neste estudo com o de foraminíferos planctônicos desenvolvido por Vicalvi (2001) para as mesmas seções como base nos zoneamentos de Ericsson \& Wollin (1968) e Vicalvi (1997), reforça a indicação da aplicabilidade deste grupo em estudos desta natureza para o Quaternário marinho brasileiro.

Por outro lado, a escassez ou mesmo ausência de estudos semelhantes inviabiliza momentaneamente a confirmação da aplicação destes resultados em uma escala maior, mesmo em nível de bacia, por isso, somente a partir da ampliação do número de seções analisadas sob este enfoque, estes resultados poderão ser corroborados e aplicados de forma mais abrangente. 


\section{AGRADECIMENTOS}

Especiais agradecimentos a M.A. Vicalvi pela disponibilização das datações com base em foraminíferos planctônicos das seções estudadas, e à PETROBRAS S.A., pela concessão dos testemunhos.

\section{REFERÊNCIAS BIBLIOGRÁFICAS}

Altenbach, A.V. \& Sarnthein, M., 1989. Productivity record in benthic foraminifera. In: W.H. Berger, V.S. Smetacek \& G. Wefer (eds.) Productivity of the ocean: Present and Past. Wiley, p. 255-270.

Barash, M.S.; Oskina, N.S. \& Blyum, N.S., 1983. Quaternary biostratigraphy and surface paleotemperatures based on planktonic foraminifers, Dee-Sea Drilling Project, Leg 72. Initial Reports DSDP, 72:849-869.

Barash, M.S.; Khusid, T.A.; Matul, A.G.; Chekhovskaya, M.P.; Biebow, H. Nürnberg, D. \& Tiedemann, R. 2008. Distribution of benthic foraminifera in upper Quaternary sediments of the Deryugin Basin (Sea of Okhotsk). Marine Geology, 48 (1):113122.

Barbosa, V.P. 2002. Sistemática, bioestratigrafia e paleoceanografia de foraminíferos do Quaternário do talude continental das bacias de Santos e Campos. Programa de Pós-graduação em Geologia, Universidade Federal do Rio de Janeiro, Tese de Doutoramento, $427 \mathrm{p}$.

Bé, A.W.; Damuth, J.E.; Lott, L. \& Free, R. 1976. Late Quaternary climatic record in western equatorial Atlantic sediment. Geological Society of America Memoir, 145:165-200.

Berggren, W.A.; Kent, D.V.; Swisher III, C.C. \& Aubry, M.-P. 1995. A revised Cenozoic geochronology and chronostratigraphy. In: W.A. Berggren, D.V. Kent, M.-P. Aubry \& J. Hardenbol (eds.) Geochronology, Time Scales and Global Stratigraphic Correlation, SEPM (Society for Sedimentary Geology), Special Pubication, 54:129-212.

Bickert, T. \& Wefer, G. 1999. South Atlantic and benthic foraminifer $\delta 13 \mathrm{C}$ deviations: implications for reconstructing the late Quaternary deep-water circulation. Deep-Sea Research, 46:437452.

Bush, A.B.G. \& Fairbanks, R.G. 2003. Exposing the Sunda shelf: tropical responses to eustatic sea level change. Journal of Geophysical Research, 108 (D15), 4446, doi:10.1029/ 2002JD003027.

Charnock, M.A. \& Jones, R.W. 1990. Agglutinated foraminifera from the Paleogene of the North Sea. In: C. Hemleben; M.A. Kaminski; W. Kuhnt \& D.B. Scott (eds.) Paleoecology, Biostratigraphy, Paleoceanography and Taxonomy of Agglutinated Foraminifera, Kluwer Academic Publishers/NATO Scientific Affairs Division, p. 433-505.

Corliss, B.H., 1979a. Taxonomy of Recent deep-sea benthonic foraminifera from the Southeast Indian Ocean. Micropaleontology, 25(1):1-29.

Corliss, B.H., 1979b. Recent deep-sea benthic foraminiferal distribution in the Southeast Indian Ocean: inferred bottom water routes and ecological implications. Marine Geology, 31(1/ 2):115-138.

Corliss, B.H., 1983. Distribution of Holocene deep-sea benthic foraminifera in the Southwest Indian Ocean. Deep-sea Research, 30(2A):95-117.

Corliss, B.H., 1985. Microhabitats of benthic foraminifera within deep-sea sediments. Nature, 314:435-438.

Corliss, B. \& Chen, C. 1988. Morphotype patterns of Norwegian Sea deep-sea benthic foraminifera and ecological implications. Geology, 16:716-719.

Corliss, B. \& Fois, E. 1991. Morphotype analysis of deep-sea benthic foraminifera from the northwest Gulf of Mexico. Palaios, 5:589-605.

CulveR, S.J. \& Buzas, M.A., 1981. Foraminifera: distribution of provinces in the Gulf of Mexico. Nature, 290:328-329.

Douglas, R.G., 1973. Benthonic foraminiferal biostratigraphy in the central North Pacific, Deep-Sea Drilling Project, Leg 17. Initial Reports DSDP, 17:607-671.

Ericsson, D.B. \& Wollin, G. 1956a. Correlation of six cores from the equatorial Atlantic and Caribbean. Deep-Sea Research, 3:104-125.

Ericsson, D.B. \& Wollin, G. 1956b. Micropaleontological and isotopic determinations of Pleistocene climates. Micropaleontology, 2(3):257-270.

Ericsson, D.B. \& Wollin, G. 1968. Pleistocene climates and chronology in deep-sea sediments. Science, 162(3859):12271234.

Fairbanks , R.G. 1989. A 17,000 year glacio-eustatic sea level record: influence of glacial melting rates on the Younger Dryas event and deep ocean circulation. Nature, 342:637-642.

Fontanier, C.; Jorissen, F.J.; Licari, L.; Alexandre, A.; Anschutz, P. \& Carbonel, P. 2002. Live benthic foraminiferal faunas from the Bay of Biscay: faunal density, composition, and microhabitats. Deep-Sea Research, 49:751-785.

Gibbard, F. \& Cohen, K.M. 2008. Global chronostratigraphical correlation table for the last 2.7 milion years. Episodes, 31(2):243-247.

Hayward, B.W; Sabaa, A. \& Grenfell, H.R. 2004. Benthonic foraminifera and the late Quaternary (last $150 \mathrm{ka}$ ) paleoceanographic and sedimentary history of the Bounty Trough, east of New Zealand. Palaeogeography, Palaeoclimatology, Palaeoecology, 211:59-93.

Herguera, J.C. \& Berger, W.H. 1991. Paleoproductivity from benthic foraminífera abundance: Glacial to postglacial change in the West-Equatorial Pacific. Geology, 19:1173-1176.

Jorissen, F.J. 1999. Benthic foraminiferal microhabitats below the sediment-water interface. In: B.K. Sen Gupta (ed.) Modern Foraminifera, Kluwer Academic Publishers, p.161-179.

Kaiho, K. 1994. Benthic foraminiferal dissolved-oxygen index and dissolved-oxygen levels in the modern ocean. Geology, 22:719722.

Keigwin Jr., L.D. 1979. Late Cenozoic stable isotope stratigraphy and paleoceanography of DSDP Sites from the East Equatorial and Central North Pacific Ocean. Earth and Planetary Science Letters, 45:361-382.

Kennett, J.P. \& Huddlestun, P. 1972. Late Pleistocene paleoclimatology, foraminiferal biostratigraphy and tephrochronology, Western Gulf of Mexico. Quaternary Research, 2:38-69.

Kohl, B.; Fillon, R.H. \& Roberts, H.H. 2004. Foraminiferal biostratigraphy and paleoenvironments of the Pleistocene Lagniappe Delta and related section, northeastern Gulf of 
Mexico. In: J.B. Anderson \& R. Fillon (eds.) Late Quaternary Stratigraphic Evolution of the Northern Gulf of Mexico Basin, SEPM (Society for Sedimentary Geology), Special Publication, 79:187-216.

Lear, C.H.; Rosenthal, Y. \& Slowey, N. 2002. Benthic foraminiferal $\mathrm{Mg} / \mathrm{Ca}$ paleothermometry: a revised core-top calibration. Geochimica et Cosmochimica Acta, 66(19):3375-3387.

Licari, L.N.; Schumacher, S.; Wenzhöfer, F.; Zabel, M. \& Mackensen, A. 2003. Communities and microhabitats of living benthic foraminifera from the tropical East Atlantic: impact of different productivity regimes. Journal of Foraminiferal Research, 33(1):10-31.

Locke, S. \& Thunell, R.C. 1988. Paleoceanographic record of the last glacial/interglacial cycle in the Red Sea and Gulf of Aden. Palaeogeography, Palaeoclimatology, Palaeoecology, 64:163187.

Loubere, P. 1991. Deep-Sea benthic foraminiferal assemblage response to a surface ocean productivity gradient: a test. Paleoceanography, 6(2):193-204.

Loubere, P. 1994. Quantitative estimation of surface ocean productivity and bottom water oxygen concentration using benthic foraminifera. Paleoceanography, 9(5):723-737.

Loubere, P. 1996. The surface ocean productivity and bottom water oxygen signals in the deep water benthic foraminiferal assemblages. Marine Micropaleontology, 28(3):247-261.

Mackensen, A.; Sejrup, H.P. \& Jansen, E. 1985. The distribution of the living benthic foraminifera on the continental slope and rise of Southwest Norway. Marine Micropaleontology, 9:275-306.

Mackensen, A.; Fütterer, D.K.; Grobe, H. \& Schmiedl, G. 1993. Benthic foraminiferal assemblages from the eastern South Atlantic Polar Front region between $35^{\circ}$ and $57^{\circ} \mathrm{S}$ : distribution, ecology and fossilization potential. Marine Micropaleontology, 22:33-69.

Mackensen, A.; Schmiedl, G.; Harloff, J. \& Giese, M. 1995. Deepsea foraminifera in the South Atlantic Ocean: ecology and assemblage generation. Micropaleontology, 41(4):342-358.

Martin, R.E.; Johnson, G.W.; Neff, E.D. \& Krantz, D.W. 1990. Quaternary planktonic foraminiferal assemblages zones of the northeast Gulf of Mexico, Colombia basin (Caribbean Sea), and tropical Atlantic Ocean: graphic correlation of microfossil and oxygen isotope datums. Paleoceanography, 5(4):531-555.

Martin, R.E.; Neff, E.D.; Johnson, G.W. \& Krantz, D.E. 1993. Biostratigraphic expression of Pleistocene sequence boundaries, Gulf of Mexico. Palaios, 8:155-171.

Martin, P.A.; Lea, D.B.; Rosenthal, Y.; Shackleton, N.J.; Sarnthein, M. \& Papenfuss, T. 2002. Quaternary deep sea temperature histories derived from benthic foraminiferal $\mathrm{Mg} / \mathrm{Ca}$. Earth and Planetary Science Letters, 198:193-209.

Ninnemann, U.S. \& Charles, C.D. 2002. Changes in the mode of Southern Ocean Circulation over the last glacial cycle revealed by foraminiferal stable isotopic variability. Earth and Planetary Science Letters, 201:383-396.

Oppo, D.W \& Fairbanks, R. G. 1987. Variability in the deep and intermediate water circulation of the Atlantic Ocean during the past 25,000 years: Northern Hemisphere modulation of the Southern Ocean. Earth and Planetary Science Letters, 86:1-15.

Ovsepyan, E. \& Ivanova, E.V. 2009. Benthic foraminifera assemblages as indicator of the paleoceanographic conditions in the Eastern Equatorial Pacific. Marine Geology, 49(1):131-140.

Peltier, W.R. \& Fairbanks, R.G. 2006. Global glacial ice volume and
Last Glacial Maximum duration from an extended Barbados sea level record. Quaternary Science Reviews, 25:3322-3337.

Piotrowski, A.M.; Banakar, V.K.; Scrivner, A.E.; Elderfield, H.; Galy, A. \& Dennis, A. 2009. Indian Ocean circulation and productivity during the last glacial cycle. Earth and Planetary Science Letters, 285:179-189.

Rangel, H.D.; Martins, F.A.L.; Esteves, F.R. \& Feijó, F.J. 1994. Bacia de Campos. Boletim de Geociências da Petrobras, 8(1):203-217.

Resig, J.M. \& Cheong, H.K. 1997. Pliocene-Holocene benthic foraminiferal assemblages and water mass history, ODP 806B, Western Equatorial Pacific. Micropaleontology, 43(4):419-439.

Schroeder, C.J. 1986. Changes in benthic foraminifer assemblages across the Holocene/Pleistocene boundary, Sites 619, 620, 621, 622, and 624, Deep Sea Drilling Project Leg 96. Initial Reports DSDP, 96:631-642.

Scott, R.W.; Combes, J.M. \& Nissen, S.E. 1998. High-Precision Chronostratigraphy of a Late Pleistocene Shelf-Edge Delta, Louisiana. Journal of Sedimentary Research, 68(4):596-602.

Sen Gupta, B.K. \& Machain-Castillo, M.L. 1993. Benthic foraminifera in oxygen-poor habitats. Marine Micropaleontology, 20(3-4):183-201.

Seto, K. 1995. Carbon and oxygen isotopic paleoceanography of the Indian and South Atlantic Oceans - paleoclimatic and paleoocean circulation. Journal Science of Hiroshima University, Series C, 10(3):393-485.

Shackleton, N.J. 1987. Oxygen isotopes, ice volume and sea-level. Quaternary Science Reviews, 6:183-190.

Shackleton, N.J. \& Opdyke, N.D. 1973. Oxygen isotope and palaeomagnetic stratigraphy of Equatorial Pacific core V28238: oxygen isotope temperature and ice volumes on a 105 year and 106 year scale. Quaternary Research, 3:39-55.

Tapia, R.; Lange, C.B. \& Marchant, M. 2008. Living (stained) calcareous benthic foraminífera from Recent sediments of Concepción, central-southern Chile $\left(\sim 36^{\circ} \mathrm{S}\right)$. Revista Chilena de Historia Natural, 81:403-416.

Thomas, E.; Booth, L.; Maslin, M. \& Shackleton, N.J. 1995. Northeastern Atlantic benthic foraminifera during the last 45,000 years: changes in productivity seen from the bottom up. Paleoceanography, 10(3):545-562.

Vergnaud-Grazzini, C.; Pujol, C. \& Duprat, J. 1983. Oxygen isotope stratigraphy and paleoclimatology of Southwestern Atlantic Quaternary sediments (Rio Grande Rise) at Deep Sea Drilling Project Site 517, Dee-Sea Drilling Project, Leg 72. Initial Reports DSDP, 72:871-884.

Vicalvi, M.A. 1997. Zoneamento bioestratigráfico e paleoclimático dos sedimentos do Quaternário superior do talude da bacia de Campos, RJ, Brasil. Boletim de Geociências da Petrobras, 11(1/ 2):132-165.

Vicalvi, M.A. 2001. Foraminíferos. In: R.O. Kowsmann; M.A. Vicaldi \& B.H. Vargas (eds.) Description and dating of piston cores from the outer shelf and slope of Santos Basin, Brazil. Rio de Janeiro, PETROBRAS/CENPES/TEGG (Comunicação Técnica $\mathrm{n}^{\circ}$ 019/01).

Waelbroeck, C.; Labeyrie, L.; Michel, E.; Duplessy, J.C.; McManus, J.F.; Lambeck, K.; Balbon, E. \& Labracherie, M. 2002. Sealevel and deep water temperature changes derived from benthic foraminifera isotopic records. Quaternary Science Reviews, 21:295-305. 
Williams, D.F.; Thunell, R.C.; Tappa, E.; Rio, D. \& Raffi, I. 1988. Chronology of the Pleistocene oxygen isotope record: 0-1.88 m.y. B.P. Palaeogeography, Palaeoclimatology, Palaeoecology, 64:221-240.

Wornardt. W.W. \& Vail, P.R. 1991. Revision of the Plio-Pleistocene cycles and their application to sequence stratigraphy and shelf and slope sediments in the Gulf of Mexico. Gulf Coast Association of Geological Societies Transactions, 41:719-744.

Received in January, 2010; accepted in May, 2010. 